\title{
Fraenkel versus Agnoli? \\ Oder: Was ist aus der „Parlamentsverdrossenheit“ der 60er Jahre für die heutige „Postparlamentarismus“-Diskussion zu lernen?
}

\author{
Uwe Thaysen und Jürgen W. Falter
}

Im letzten Heft dieser Zeitschrift (ZParl) fand sich ein Hinweis auf ihren Beginn: In seinem Aufsatz über Johannes Agnoli und dessen illiberale bis antidemokratische Parlamentarismuskritik benannte Wolfgang Kraushaar ${ }^{1}$ ein Motiv für die Gründung der ZParl vor bald schon 40 Jahren $^{2}$. Anlass genug, in eben dieser Zeitschrift Genaueres nachzureichen.

Kraushaar hatte seine Darlegung der Nähe von Agnoli und dessen Werk zum italienischem Faschismus zuvor auf einer Tagung des Deutsch-Italienischen Zentrums Villa Vigoni vorgetragen und - wie nicht anders zu erwarten - heftige Reaktionen erfahren ${ }^{3}$. In einer der Repliken hieß es, entscheidend für die Wertung von Agnoli sei nicht der Nachweis „einer ungewollten und zeitbedingten Nähe [zur präfaschistischen beziehungsweise faschistischen Parlamentarismuskritik], sondern die Frage, ob diese Nähe erlebt wurde, erlebbar war oder nicht “ ${ }^{\star}$. Zu den Stärken von Johannes Agnoli gehörte genau dies: dass er „erlebbar“ war, dass er kaum jemanden „kalt ließ“. So haben ihn auch die Verfasser dieser Reminiszenz erfahren, die 1967/1968 zusammen mit ihm und anderen um eine studentisch betriebene politikwissenschaftliche Zeitschrift bemüht waren. Daraus ergibt sich für uns nicht nur der Anlass zu biografischem Nachtrag hinsichtlich der Person Agnoli, sondern auch die Notwendigkeit der Auseinandersetzung mit seinem Wirken bis heute.

Erstens möchten wir auf den noch keineswegs systematisch für alle Universitäten der Bundesrepublik erschlossenen studentischen Beitrag zur Politikwissenschaft im Nachkriegsdeutschland aufmerksam machen. Zweitens möchten wir den Rang beleuchten, den damals - im Unterschied zu heute? - das Thema „(Anti-)Parlamentarismus“ innehatte. Drittens stellen wir einige Hypothesen auf, davon ausgehend, dass die Diskussion um Anspruch und Wirkung der ,Achtundsechsziger' keineswegs abgeschlossen ist, sondern durchaus noch etwa aus Anlass ihres ,Vierzigjährigen'? - den Stoff eines bislang nicht wirklich offen ausgetragenen Historikerstreites in sich birgt, Überlegungen sowohl zur damaligen als auch zur heutigen Diskussion des (Post-)Parlamentarismus, der (Post-)Demokratie und der Rolle des Staates als des Garanten ernstzunehmender demokratischer Spielregeln. Für uns ist evident:

1 Wolfgang Kraushaar, Agnoli, die APO und der konstitutive Illiberalismus seiner Parlamentarismuskritik, in: ZParl, 28. Jg. (2007), H. 1, S. $160-180$.

2 Ebenda, S. 167 und 173; siehe dazu, gewissermaßen als Merkposten in eigener Sache, auch den entsprechenden Hinweis im Editorial desselben Heftes.

3 Siehe den Bericht von Rainer Blasius über diese Tagung, „Seitenwechsel und Veränderung “, in: FAZ vom 12. Dezember 2006, S. 12. Siehe dazu auch Leserbriefe der Empörung von Frank Deppe und Jürgen Frese in derselben Zeitung vom 21. Dezember 2006, S. 8 sowie von Barbara Görres Agnoli, der Witwe Johannes Agnolis, ebenfalls in der FAZ vom 21. Januar 2007, S. 7. Zum Parlamentarismusverständnis des damaligen SDS-Theoretikers, Frank Deppe, heute Professor an der Universität Frankfurt am Main, siehe Frank Deppe, Parlamentarismus - Parlamentarische Aktion - Sozialistische Politik, in: Neue Kritik, Nr. 44, November 1967, S. 48 - 67.

4 Zitiert nach dem Bericht von Rainer Blasius, a.a.O. (Fn. 3); Zusatz in Klammern von den Autoren dieses Beitrages. 
Im heutigen Verdrossenheitsdiskurs - ob Demokratie- ${ }^{5}$, Institutionen- ${ }^{6}$, Parteien-7 ${ }^{7}$, Parlaments ${ }^{8}{ }^{8}$, Politik- ${ }^{9}$, Politiker- ${ }^{10}$ oder Staatsverdrossenheit ${ }^{11}$ - haben wir es mit einem politisch riskanten Gemisch zu tun, einem intellektuellen Knäuel von alten und neuen Begründungen, das dringend der Entwirrung bedarf. Zu weiterem Diskurs über damalige Autoren und Akteure sowie zur dringend angezeigten Erforschung der Unterschiede der Parlamentsund Demokratieverdrossenheit beider Epochen sei hiermit - entlang einer Zeitschriftengeschichte in der Geschichte - nachdrücklich ermuntert.

\section{DER POLITOLOGE - ein studentischer Beitrag zur Politikwissenschaft}

Apodiktisch heißt es in Wilhelm Bleeks detailgetreuer Monografie zur Geschichte der deutschen Politikwissenschaft: „Von Anfang an spielte in ... Forschung und Lehre der bundesdeutschen Politikwissenschaft [der] Parlamentarismus eine wichtige Rolle. "12 Diese Feststellung gilt auch bezüglich der Aufmerksamkeit, die einzelne Aspekte der damals aktuellen Parlamentspraxis in der Studentenschaft des Faches jener Tage fanden. Im Allgemeinen aber, so konstatiert Gerhard Lehmbruch zutreffend, begegne man in den Darstellungen des (Neu-)Beginns der Disziplin nach 1945 „einer eigentümlichen ,top-down’ Perspektive“; aus dieser - auf die „Gründungsväter“ des Faches beschränkten - Sicht werde der studentische Beitrag zur Neu(be)gründung der Politologie völlig übersehen. Dagegen wünscht Lehmbruch, „dass die Gründung der westdeutschen Politikwissenschaft insbesondere vor dem

5 Vgl. dazu die Bestandsaufnahme der Diskussion bei Hubertus Buchstein / Dirk Jörke, Das Unbehagen an der Demokratietheorie, in: Leviathan, 31. Jg. (2003), S. 470 - 495.

6 Das seit 2006 neue Redaktionsteam von Parliamentary Affairs konstatiert in seinem ersten Editorial einen "trend of alienation from, apathy about and cynicism toward representative institutions, both in Britain and elsewhere", siehe ebenda, 59. Jg. (2006), S. 7. Vgl. für Deutschland jüngst mit sechs umfassenden Artikeln und als Leitthema bemerkenswert breit aufgemacht: „Das Volk hört weg. Viele Bürger zweifeln an der Demokratie, obwohl sie keine Extremisten sind. Gerät unser System in Gefahr?", in: Die Zeit vom 18. Januar 2007, S. 1 - 5.

7 Peter Lösche, Parteienverdrossenheit ohne Ende? Polemik gegen das Lamentieren deutscher Politiker, Journalisten, Politikwissenschaftler und Staatsrechtler, in: ZParl, 26. Jg. (1995), H. 1, S. 149 $-159$.

8 Svein S. Andersen / Tom R. Burns, The European Union and the Erosion of Parliamentary Democracy: A Study of Post-parliamentary Governance, in: Svein S. Andersen / Kjell E. Eliassen (Hrsg.), The European Union: How Democratic Is It?, London 1996, S. 227 - 251; Arthur Benz, Postparlamentarische Demokratie? Demokratische Legitimation im kooperativen Staat, in: Michael Th. Greven (Hrsg.), Demokratie - eine Kultur des Westens? 20. Wissenschaftlicher Kongreß der Deutschen Vereinigung für Politische Wissenschaft, Opladen 1998, S. 201 - 220.

9 Brigitte Geißel, (Un-)Geliebte Profis? - Politikerverdrossenheit und Politikerprofessionalität. Daten von der lokalen Ebene, in: ZParl, 37. Jg (2006), H. 1, S. 80 - 96.

10 Ebenda.

11 Im Falle der Beschwörung von „Staatsverdrossenheit“ handelt es sich zumeist sowohl um die weithin geführte Klage über generell mangelnde staatliche Problemlösungskapazität aufgrund der Einbindung des Staates in korporatistische sowie internationale bis globale Arrangements als auch um das Beklagen der Ergebnisse dennoch getroffener konkreter einzelner Policy-Entscheidungen. So oder so wird „Staatsversagen“ moniert: mit dem Argument der „Reformunfähigkeit“ oder mit dem der „Überregulierung“.

12 Wilhelm Bleek, Geschichte der Politikwissenschaft in Deutschland, Darmstadt 2001, S. 295; Hervorhebung im Original. 
Hintergrund der politischen Bewusstseinslage der Studentenschaft nach dem Zweiten Weltkriege" gesehen werde ${ }^{13}$, und er verdeutlicht seine Ermahnung vornehmlich am Beispiel des Tübinger Seminars für Politikwissenschaft, aus dessen studentischem „Arbeitskreis für Politikwissenschaft“ er selbst und eine ansehnliche Reihe weiterer Professoren hervorgingen - darunter auch Johannes Agnoli, der spätere „Wortführer der ,undogmatischen’ Marxisten am Otto-Suhr-Institut" ${ }^{14}$ der FU Berlin.

Mit guten Gründen wurde in den 1950er und 1960er Jahren davon ausgegangen, dass „Westberlin nicht nur zum Vorposten der westlichen Welt, sondern auch zur Vorhut bei der [Entwicklung einer] bundesdeutschen Politikwissenschaft [als Demokratiewissenschaft] wurde" 15 . Ganz so sahen es jene damaligen Politologiestudenten, die sich vor genau 50 bis fast genau 40 Jahren als Herausgeber einer eigenen Zeitschrift ihrer Disziplin, des anfänglich so genannten POLITOLOGEN, bewähren wollten.

In den Jahren 1957 bis 1968 veröffentlichte die Fachschaft der Deutschen Hochschule für Politik, seit dem Wintersemester 1959/60 Fachschaft Politologie am Otto-Suhr-Institut (OSI) der FU-Berlin, im POLITOLOGEN Informationen und Reflexionen über ihre Disziplin, namentlich zur Theorie des Parlamentarismus ${ }^{16}$. Das Zeitschriftenprojekt gedieh sowohl quantitativ als auch qualitativ beständig, fand aber nichtsdestoweniger 1968 ein jähes Ende. An seine Stelle traten dann gleich zwei Zeitschriften: „Sozialistische Politik“

13 Gerhard Lehmbruch, Demokratieforschung und Demokratieerziehung in der Nachfolge Theodor Eschenburgs; Auszüge, wiedergegeben in: Barbara Görres Agnoli, Johannes Agnoli. Eine biografische Skizze, Hamburg 2004, S. $161 \mathrm{f}$.

14 Ebenda, S. 171. In seiner Rezension der biografischen Skizze von Barbara Görres Agnoli, a.a.O. (Fn. 13) fasst Rudolf Walther den politischen Lebensweg von Johannes Agnoli in die Titelzeile: „Vom Bewunderer Mussolinis zum Wortführer der Apo“, in: Die Zeit vom 31. Dezember 2004. Walther eröffnet seine Rezension mit der Feststellung: „Wenn es ein Buch gegeben hat, das man als Bibel der außerparlamentarischen Opposition (Apo) bezeichnen kann, dann war es ,der Agnoli', das heißt das Buch mit dem Titel Die Transformation der Demokratie." Agnolis Biografie ist ein beredtes Beispiel für die Ausnahmen vom Allgemeingültigkeitsanspruch des Mannheimschen Generationenkonzeptes (siehe zu dessen sonstiger Übertragbarkeit auf die Kohorten politikwissenschaftlicher Universitätslehrer Wilhelm Bleek, a.a.O. (Fn. 12), S. 368): Den Gründungsvätern der Politologie, wie Ernst Fraenkel (1898 bis 1975) teils sogar noch deutlich vor dem Ersten Weltkrieg geboren, mit ihren Erfahrungen des Scheiterns der ersten deutschen Demokratie, des Nationalsozialismus und des Zweiten Weltkrieges ist Agnoli (Jahrgang 1925) generationsmäßig nicht zuzurechnen; deren ersten Schülern aus der unmittelbaren Nachkriegszeit seit 1945 ist er auf Grund seiner geographischen (italienischen) und politischen Herkunft ebenso wenig zuzuordnen wie der deutschen Flakhelfergeneration; der dann folgenden Generation der ,Studentenbewegung' war er mit seinen immerhin schon 40 Jahren zwar altersmäßig bereits entwachsen, gleichwohl wurde er einer ihrer Exponenten, für viele ein gläubig bewunderter Mentor. Es wurde die Rolle seines weiteren Lebens bis zu seinem Tode im Jahre 2003.

15 Wilhelm Bleek, a.a.O. (Fn. 12), S. 284, Text in Klammern von uns hinzugefügt.

16 Nach heutigem Verständnis von Empirie lag zurzeit des POLITOLOGEN noch zu wenig einschlägiges, in sich methodologisch stringent vergleichbares Material über die Anfangszeit der Bundesrepublik vor. Auch daraus erklärt sich die anfängliche Konzentration der Gründergeneration auf die Theorie (anstelle der Empirie) des Parlamentarismus. Zunächst musste es um die Maßstäbe gehen, die an die Empirie anzulegen seien. Dieser Zusammenhang erklärt unter anderem, wie es kommen konnte, dass ausgerechnet einem so scharfen Beobachter der Wirklichkeit wie Ernst Fraenkel, einem Kritiker auch der als solcher von ihm durchaus benannten Fehlentwicklungen der sechziger Jahre, der massive Vorwurf der Empirieferne, um in heutiger Sprache zu bleiben: der Wirklichkeitsverweigerung, gemacht wurde. Siehe dazu unten Fn. 39. 
(SoPo) sowie „Probleme des Klassenkampfes“ (PROKLA) einerseits, und, nicht zuletzt in Reaktion auf diese beiden, die „Zeitschrift für Parlamentsfragen“ (ZParl) andererseits. Bei alledem spielte Johannes Agnoli keine unbedeutende Rolle. Zumal im Universitätsalltag gab er sich als ein Verfechter von Emanzipation und Partizipation auf allen Ebenen, in seinem akademischen Umfeld als vehementer Anwalt studentischer Autonomiebestrebungen aus. Umso aufschlussreicher ist, was unter seiner Mitwirkung aus dem POLITOLOGEN wurde, der, damals in studentischer Regie herausgegeben, Themen der Demokratietheorie, darunter dezidiert auch Themen des Parlamentarismus, gewidmet war.

Die ersten Ausgaben des POLITOLOGEN erschienen 1957: hektografiert, 20 bis 30 Seiten stark, bis 1959 im Din A 5-Format geheftet, bis 1961 schreibmaschinenschriftlich. Inhaltlich zunächst auf die Selbstfindung des Faches vor Ort konzentriert und daher spöttisch als „Hauspostille“ der Berliner Politologen apostrophiert, bezogen sich die weiteren Hefte zunehmend auch auf die westdeutsche und die internationale westliche Politikwissenschaft. Verändertes Selbstverständnis und wachsendes Selbstbewusstsein der Redaktion fanden ihren Niederschlag in fortlaufenden Namensänderungen ihrer Zeitschrift, die bis 1970 über Westberlin hinaus ein veritables wissenschaftliches Periodikum des Faches darstellte.

Bis 1966 erschien die Zeitschrift als DER POLITOLOGE - von 1959 bis zum letzten Heft des Jahres 1965 in Din A 4-Format, seit 1962 in Hochglanz eingeschlagen und auch im Inneren bebildert. Deutliche Veränderungen, „revolutionäre Ideen“ gar, wurden im Editorial der Dezemberausgabe 1965 angekündigt und dann auch wirklich mit dem Heft Nr. 19 vom April 1966 in die Tat umgesetzt. Wissenschaftlich selbstgewiss verkündete die neue studentische Redaktion im Editorial des Heftes 19: „Ohne Bilder und Hochglanzumschlag glauben wir unserer neuen Konzeption besser gerecht zu werden ..." Die programmatische Neuorientierung der Redaktion wurde eingeleitet von Wolff-Dieter Eberwein (verantwortlich), Monika Bogus und Jürgen Falter. Von hier an erschien die Zeitschrift in neuer Zählung, verlässlich als vierteljährliches Periodikum. Eine Unterzeile des inzwischen etablierten Zeitschriftentitels signalisierte die neue Akzentuierung: „Berliner Zeitschrift für Politische Wissenschaft“; nur wenig später ließ eine Veränderung auch des Impressums den gesteigerten Ehrgeiz der neuen Redaktion erkennen: Vom Heft 23 an firmierte nicht mehr die Fachschaft allein als Herausgeber, sondern es waren nun die Professoren Richard Löwenthal und Gilbert Ziebura hinzugekommen. Von der Nummer 22 an (8. Jg., 1967) war der eine Autor dieser Reminiszenz (Jürgen Falter) als Chefredakteur, später als Mitherausgeber, ausgewiesen; der andere (Uwe Thaysen), wie damals Johannes Agnoli Assistent an der FU, fungierte, wie dieser, als (zusammenarbeitendes) Mitglied (ab H. 23 als Mitglied des „Redaktionsbeirates“). Vom Heft 23 an gehörte neben Jürgen Domes, Franz Ansprenger und Ekkehard Krippendorf auch Winfried Steffani dem neu eingerichteten „Redaktionsbeirat“ an. Jetzt hieß es auf der Titelseite nur noch, dass die Zeitschrift am OSI der FU herausgegeben werde, und mit dem 9. Jahrgang (1968) waren schließlich fast alle Hinweise auf das ursprüngliche Selbstverständnis des POLITOLOGEN getilgt: Seit der Nr. 25 (H. 1/1968) bis zu ihrer letzten, der 29. Ausgabe (H. 4/1968), firmierte die Zeitschrift stolz als „Berliner Zeitschrift für POLITOLOGIE“, herausgegeben am OSI der FU Berlin.

Der gesteigerte Anspruch führte in zweierlei Schwierigkeiten: Je renommierter die gewonnenen Autoren, umso geringer wurde der Anteil studentischer Beiträge. Das neue, auf akademische Professionalisierung bedachte Äußere, vor allem aber der tatsächlich gesteigerte wissenschaftliche Anspruch erschwerten das Anzeigengeschäft, über welches sich die nach wie vor im Wesentlichen von Studierenden gemachte - Zeitschrift finanzierte. Den- 
noch wurde eine Auflagenhöhe erreicht, die derjenigen der „Politischen Vierteljahresschrift“ (PVS), dem professionell - um nicht zu sagen professoral - organisierten „Zentralorgan“ der Zunft, nahezu gleichkam.

Die einst hausinterne Selbstvergewisserung trat vom Heft 19 an immer deutlicher in den Hintergrund gegenüber dem Bestreben, „über Entwicklungen auf unserem Fachgebiet“ zu informieren und „Analysen des Tagesgeschehens“ anzubieten. Aus den Beiträgen (später) renommierter Fachvertreter wie Niels Diederich, Dieter Grosser, Hartmut Jäckel, Georg Kotowski, Kurt Sontheimer, Rudolf Wildenmann und anderer Autoren der zweiten und dritten Generation künftiger Lehrstuhlinhaber ließe sich eine noch heute weithin gültige Anthologie zur Einführung in die Politikwissenschaft zusammenstellen. Schon die Titel ihrer Beiträge zeigen an, dass sich das Politologieverständnis dieser Autoren deutlich unterschied von demjenigen zweier strikt ökonomisch-soziologisch appellierender Aufsätze aus der Feder Johannes Agnolis in den letzten Nummern der Berliner Zeitschrift für POLITOLOGIE ${ }^{17}$. Auch lässt ein einziger Blick auf die Themen dieser Hefte erkennen, dass damals politisch aktuelle Streitthemen, insbesondere die mit Vehemenz einsetzende Diskussion um Totalitarismus- und Faschismustheorien sowie Rätedemokratie, deutlich in den Vordergrund traten. Dennoch blieb der bis dahin geübte wissenschaftliche und politische Pluralismus bis 1969 das Leitmotiv der Herausgeber und mindestens der Mehrheit der Redak-tion.

\section{Agnoli versus Fraenkel, Fraenkel versus Agnoli?}

Besondere Beachtung der Parlamentarismusforschung verdienen heute - nach 40 Jahren immer noch beziehungsweise wieder (!) - drei Aufsätze, die 1967 und 1968 im POLITOLOGEN veröffentlicht wurden, einer von Ernst Fraenkel ${ }^{18}$ sowie die zwei erwähnten von Johannes Agnoli zur Faschismusdiskussion. Inhaltlich liegen Welten zwischen den Ansatzund Zielpunkten der beiden Autoren zum Parlamentarismus. Zwar spiegelt dies die Liberalität der damaligen Redaktion wider; Lesern, die mit den zu jener Zeit am OSI heftig geführten Diskussionen weniger vertraut sind, dürften gleichwohl die später von Agnoli selbst und seiner Witwe gegebenen Hinweise darauf kaum verständlich sein, dass ausgerechnet der dezidierte Parlamentarismusaufklärer und Verfechter parlamentarischer Regierungsweise Ernst Fraenkel seine „schützende Hand“19 über den expliziten Parlamentsverächter Agnoli gehalten habe - nicht nur als es um dessen Einstellung am OSI ging, sondern auch später noch: „Über Ernst Fraenkels Förderung hat sich Agnoli immer gewundert.“20

17 Johannes Agnoli, Zur Faschismusdiskussion (I), in: Berliner Zeitschrift für POLITOLOGIE, 9. Jg. (1968), Nr. 2, S. 24 - 33; ders., Zur Faschismusdiskussion (II), ebenda, 9. Jg. (1968), Nr. 4, S. $32-49$.

18 Ernst Fraenkel, Ursprung und politische Bedeutung der Parlamentsverdrossenheit, in: DER POLITOLOGE, 8. Jg. (1967), H. 2, S. 17 - 25. Zuerst erschienen in: Integritas, Geistige Wandlung und Menschliche Wirklichkeit, Tübingen 1966, S. 244 ff.; später aufgenommen in seine wiederholt aufgelegte Aufsatzsammlung „Deutschland und die westlichen Demokratien“. Der Vorabbeziehungsweise Nachdruck damals einschlägiger Artikel war ein Versuch der Zeitschrift DER POLITOLOGE beziehungsweise der Berliner Zeitschrift für POLITOLOGIE, die Studenten jener noch Kopierer-unzugänglichen Tage zur Lektüre auf dem Stand der damaligen wissenschaftlichen Diskussion zu verhelfen.

19 Siehe Barbara Görres Agnoli, a.a.O. (Fn. 13), S. 65.

20 Ebenda. 
Fraenkel, ein remigrierter "Trotzjude“, wie er selbst in Anlehnung an Theodor Herzl betonte, hat in seinen Lehrveranstaltungen immer wieder Differenzierungen des italienischen Faschismus sowie die in seinen Augen schwerwiegenden Unterschiede des deutschen zum italienischen Faschismus hervorgehoben. Insbesondere die Tatsache, dass der italienische Faschismus anfänglich nicht antisemitisch begründet und später nicht so perfektioniert barbarisch wie der deutsche war, mag ihn bewogen haben, so unsere erste Hypothese, Agnolis exzellenten Kenntnisse solcher Differenzierungen zu schätzen. Dafür lassen sich bei Agnoli Belegstellen wie die folgende zitieren: „Richtig ist allerdings, daß der Antisemitismus keinen nennenswerten Faktor innerhalb des italienischen Faschismus bildete - weder ideologisch noch sozial: ein Sachverhalt, der nicht immer genügend reflektiert wird, so daß die Gleichung Faschismus - Antisemitismus sich zu schnell einstellt und zu schiefen Urteilen sowohl über Personen wie über Bewegungen führt." 21

Außerdem dürfte Fraenkel, selbst ehedem „sozialistisch-klassenkämpferisch“ engagiert, in Agnoli, so unsere zweite Hypothese, den Philosophen, zumal den Linkshegelianer zu würdigen gewusst haben.$^{22}$

Der gewagteste unserer Erklärungsversuche für Fraenkels ansonsten schwer verständliche „Förderung“ ist, dass die von ihm diagnostizierte „Doppelstaatlichkeit“ des Nationalsozialismus ${ }^{23}$ bezüglich der Rolle des Staates Parallelen zu den von Agnoli konstatierten „Kontingenzien" 24 der bürgerlichen Organisation von Herrschaft aufweist. In dieser Doppelbödigkeit staatlicher Herrschaft, in Agnolis Kenntnis der „Varietät“ auch „der Masken eines faschistischen Parlamentarismus“ ${ }^{25}$, seinem Insistieren auf den ,institutionell und ideologisch variablen Typen des Parlamentarismus“ ${ }^{26}$ bis hin zur Verbindung von „totalem Herr-

21 Johannes Agnoli, Faschismusdiskussion II, a.a.O. (Fn. 17), S. 42. Die beiden von ihm in der POLITOLOGIE veröffentlichten Beiträge zur Faschismusdiskussion stellen einen Rezensionsessay in Fortsetzung dar, der auch heute noch zur Pflichtlektüre der Beschäftigung mit dem Faschismus, insbesondere mit der Rezeption der Faschismustheorie in Deutschland, zu rechnen ist.

22 Siehe Alexander von Brünneck, Hubertus Buchstein und Gerhard Göhler, die Herausgeber des einschließlich des Registers auf sieben Bände angelegten Gesamtwerkes von Ernst Fraenkel in ihrem Vorwort zu den Gesammelten Schriften, Bd. I, 1. Auflage, Baden-Baden 1999, S. 12; siehe auch die Charakterisierung der Beiträge Ernst Fraenkels der 1920er und 1930er Jahre mit „starken Einschlägen eines nichtkommunistischen Marxismus“ bei Rudolf Wolfgang Müller, „....wenn es morgens um 6 klingelt, war es der Milchmann" - Ernst Fraenkel und die West-Berliner Studentenbewegung 1967, in: Hubertus Buchstein / Gerhard Göhler (Hrsg.), Vom Sozialismus zum Pluralismus. Beiträge zu Werk und Leben Ernst Fraenkels, Baden-Baden 2000, S. 99. Zur Kapitalismuskritik im ersten der Hauptwerke von Fraenkel, dem Doppelstaat, siehe Alexander v. Brünneck, Ernst Fraenkels Urdoppelstaat von 1938 und der Doppelstaat von 1941/1974, in: ebenda, S. 29 - 42, besonders S. 32.

23 In seinem berühmten Werk, Der Doppelstaat, als Nachdruck neu ediert in: Ernst Fraenkel, Gesammelte Schriften, Bd. II, Baden-Baden 1999, S. 33 - 226 als deutsche Erstübersetzung der englischen Urfassung des ,Dual State'. Zur wechselvollen Geschichte dieses Werkes siehe von Alexander v. Brünneck, Ernst Fraenkels Urdoppelstaat, a.a.O. (Fn. 22).

24 Johannes Agnoli, Faschismusdiskussion I, a.a.O. (Fn. 17), S. 27.

25 Ebenda, S. 28.

26 Ders., Faschismusdiskussion II, a.a.O. (Fn. 17), S. 42. Immer war in seinen Lehrveranstaltungen zu erfahren, wie sehr Fraenkel die materialen Analysen Hermann Hellers, eines anderen Kenners des italienischen Faschismus, verehrte. Mit gutem Grund; siehe Hermann Heller, der selbst einige Zeit in Italien gelebt hatte: Europa und der Faschismus, zuerst veröffentlicht 1929, neu herausgegeben in: ders., Gesammelte Schriften, Bd. II, 2. Auflage, Tübingen 1992, S. 463 - 611. Was Heller, S. 467 ff., damals - teils erschrocken, wie es scheint - in Auseinandersetzung mit Karl Marx, Carl Schmitt und den Apologeten des italienischen Faschismus seiner Zeit schrieb, nimmt 
schaftsanspruch und Konstitutionalität" ${ }^{\text {"27, }}$, so unsere dritte Hypothese, liegt eine gewisse Faszination, die Fraenkel in seiner Einschätzung des Nachwuchswissenschaftlers am OSI bestimmt haben könnte.

Schließlich mag Fraenkel sich, so unsere vierte Hypothese, als jener Pluralismustheoretiker gesehen haben, der auch in der Praxis der universitätsinternen Politik für Minderheitspositionen und Vielfalt gerade zu stehen hatte ${ }^{28}$ - mindestens bis zu seiner tiefgreifenden Verbitterung über die Entwicklung der „Studentenbewegung“ und die APO ${ }^{29}$ seit 1967.

Über Fraenkels tatsächliche Bewertung der beiden Aufsätze von Agnoli zur Faschismusdiskussion in der Berliner Zeitschrift für POLITOLOGIE wie, vor allem, Fraenkels Bewertung von Agnolis Kampfschrift ${ }^{30}$ „Transformation der Demokratie“31 ist den Verfassern dieser Zeilen nichts Explizites bekannt geworden. Außerhalb jeden Zweifels steht für uns jedoch, dass Fraenkels pädagogisch geradezu missionarisches Eintreten für ein generell aufgeklärtes „empirisches“ Verständnis des Parlamentarismus und für eine im Grundsatz wohlgesinnte kritische Betrachtung des erfahrbaren bundesdeutschen Parlamentarismus ganz und gar nicht in Einklang zu bringen ist mit Agnolis Klassifizierung der entscheidenden Parlamentsfunktion im Kontext seiner für die Bundesrepublik vorgetragenen „Involutionsthese“32. Er scheint deshalb durchaus in Schutz zu nehmen zu sein vor jenen heutigen Kritikern seiner Kritiker, die ihn als antiparlamentarischen Revolutionär ${ }^{33}$ offenbar nicht (mehr) ernstnehmen wollen und insoweit verharmlosen; Agnoli, den auch wir als persönlich liebenswürdigen Dialektiker kennen lernten, amüsierte derlei zu seinen Lebzeiten.

sich bei erneuter Lektüre aus wie heute gültige Zurückweisungen gegenwärtiger Argumente des Parlamentsverdrusses: „Der Parlamentarismus ... befindet sich nur deshalb in der Krise, weil die vorausgesetzte Wert- und Willensgemeinschaft, welche die den Parteiungen für die gewaltlose parlamentarische Diskussion unentbehrliche gemeinsame Diskussionsbasis bietet, weitgehend fehlt. Und tatsächlich sind die sozialen Gegensätze in dem gleichen Augenblicke nicht mehr demokratisch zu organisieren, in dem dem politischen Gegner die gemeinsame Diskussionsgrundlage nicht zugebilligt wird." (S. 467) Siehe zur offenen Verhöhnung des Parlaments, besonders der Abgeordneten unserer Tage durch die derzeitige deutsche Funktionselite die Belege einschließlich der Zitate in den Beiträgen der „Zeit“ vom 18. Januar 2007, a.a.O. (Fn. 6).

27 Johannes Agnoli, Faschismusdiskussion II, a.a.O. (Fn. 17).

$28 \mathrm{Zu}$ Fraenkels „Kämpfernatur“ siehe Winfried Steffani, Ernst Fraenkel als Persönlichkeit, in: $H u$ bertus Buchstein / Gerhard Göhler (Hrsg.), a.a.O. (Fn. 22), S. 134 f.: „Als politischer Mensch forderte er [Fraenkel] von jedem zugleich die Fähigkeit und den Mut zur persönlichen politischen Grundentscheidung. Bei ihm hieß dies Einsatz für persönliche Freiheit in Verantwortung, Recht für jeden und soziale Gerechtigkeit ohne Ausnahme."

29 Siehe Rudolf Wolfgang Müller, a.a.O. (Fn. 22); ferner Winfried Steffani, a.a.O. (Fn. 28), S. 139 ff.

30 Unter dem bei Karl Marx entlehnten Motto: „Man muss diese versteinerten Verhältnisse dadurch zum Tanzen zwingen, daß man ihnen ihre eigene Melodie vorsingt!“

31 Zuerst erschienen im Voltaire Verlag, Berlin 1967.

32 Siehe hierzu den in Fn. 1 genannten Beitrag von Wolfgang Kraushaar. Agnolis Definition der Involution: „Involution’ bildet den korrekten Gegenbegriff zu Evolution. Der Terminus hat sich in der politischen Sprache der romanischen Länder eingebürgert und bezeichnet sehr genau den komplexen politischen, gesellschaftlichen und ideologischen Prozeß der Rückbildung demokratischer Staaten, Parteien, Theorien in vor- oder antidemokratische Formen. "Hier zitiert nach der EVA-Ausgabe der „Transformationsschrift“: Die Transformation der Demokratie (Fn. 14 und 32), S. 10. Zur Entstehungsgeschichte der Schrift siehe Wolfgang Kraushaar, a.a.O. (Fn. 1).

33 Siehe zur gleichermaßen kritischen Würdigung des Revolutionärs Agnoli grundlegend schon Gerhard A. Ritter, Der Antiparlamentarismus und Antipluralismus der Rechts- und Linksradikalen, in: APuZ, B 34 (1969), S. 3 - 33, besonders S. $14-18$. 


\section{Fraenkel, Agnoli und die gegenwärtige Rede vom „Postparlamentarismus“}

Zwar ermahnt Agnoli wie Fraenkel (wenngleich dieser mit anderen Worten als jener), die „Kontingenzien“ alles dessen nicht zu übersehen, was „im besonderen wirtschaftlichen, politischen, kulturellen Fall Faschismus sein kann“34; zwar setzt Agnoli Parlamentarismus und Faschismus in der Quintessenz seiner beiden genannten Beiträge zur Faschismusdiskussion $^{35}$ wie in seinem Traktat "Transformation der Demokratie“ in eins. Genau dabei ist er jedoch nicht bereit oder außer Stande, jene „Kontingenzien“ tatsächlich zu berücksichtigen, die Ernst Fraenkel ein Jahr zuvor im POLITOLOGEN ${ }^{36}$ als unverzichtbar für jede politologische Parlamentarismusanalyse herausstellte. Fraenkel beginnt seinen grundlegenden Aufsatz zur Parlamentarismustheorie und zur Geschichte des westdeutschen Parlamentarismus mit der Prägung des Begriffes „Parlamentsverdrossenheit“ in einem Kontext, wie er aktuell bezüglich der Bundestagswahl 2005 kaum anders interpretiert wird:

„Anläßlich der Bundestagswahl des Jahres 1965 machte sich ein weit verbreitetes vages politisches Unbehagen geltend, das in einer nur unscharf artikulierten Kritik an dem Bonner parlamentarischen Betrieb in Erscheinung trat, und das im folgenden als ,Parlamentsverdrossenheit' bezeichnet werden soll. Gerade, weil es sich um Vorgänge handelt, die sich vornehmlich im kollektiven Unterbewusstsein abspielen, ist es umso notwendiger, sie in breiter Öffentlichkeit zu erörtern. Wir sollten uns rechtzeitig einer Art politologischer Psychoanalyse unterziehen, um zu verhindern, daß wir abermals einer oder gar mehreren politischen Neurosen zum Opfer fallen. Der 19. September 1965 war das genaue Gegenteil einer Protestwahl. Er war trotz allen unterschwelligen Mißbehagens der Ausdruck eines zum mindesten äußeren Wohlbehagens. Die Bundestagswahl 1965 stellt ein mißmutiges Bekenntnis zu unserer Wohlfahrtsdemokratie dar. ${ }^{\text {"37 }}$

War die Bundestagswahl 2005 etwas prinzipiell anderes als „ein mißmutiges Bekenntnis zu unserer Wohlfahrtsdemokratie“? Wer wollte heutige Parlamentsverdrossenheit leugnen?38

Unter Zugrundelegung der - ihrerseits klassisch gewordenen - Dichotomie von klassisch „rationalem“ und klassisch „empirischem“39 Parlamentsverständnis plädiert Fraenkel für

34 Johannes Agnoli, Faschismusdiskussion I, a.a.O. (Fn. 17), S. 27 f.

35 Siehe Fn. 17.

36 Ernst Fraenkel, Ursprung und politische Bedeutung der Parlamentsverdrossenheit, a.a.O. (Fn. 18).

37 Ebenda, S. 17.

38 Siehe dazu die notwendigsten Anmerkungen im Editorial des Heftes 1/2007 dieser Zeitschrift.

39 Kai Arzheimer, Politikverdrossenheit, Bedeutung, Verwendung und empirische Relevanz eines politikwissenschaftlichen Begriffes, Wiesbaden 2002, hat sich umfassend kritisch, dabei, S. 36 39, auch bezüglich Ernst Fraenkels vielzitiertem Aufsatz zur Parlamentsverdrossenheit, mit der analytischen (Un-)Tauglichkeit der Verdrossenheitsvokabel auseinandergesetzt. Meines Erachtens (Uwe Thaysen) ist Arzheimers Kritik, derzufolge Fraenkel auf eine „empirische Absicherung der von ihm postulierten Kausalbeziehungen verzichtet" (S. 38), erstens unzutreffend, insofern Fraenkel mächtige (heutigen „empirischen“ Zahlen der Meinungsforschung gewiss ebenbürtige) historische Evidenz wie die Wirkung der antidemokratischen Verdrossenheit zu Zeiten der Weimarer Republik heranzieht. Dies ist, zweitens, umso mehr gegen Arzheimers Einwand zu betonen, als Fraenkels Anspruch auf Empirie in seinem Aufsatz zur Parlamentsverdrossenheit kategorial, also gar nicht im Sinne einer hier und jetzt in diesem Aufsatz einzulösenden empirischen Gewichtung (damals gegenwärtiger „Parlamentsverdrossenheit") erhoben wird. Arzheimers Kritik ist dazu angetan, Fraenkel einmal mehr zu Unrecht eine immer wieder - mehr implizit als ausgesprochen prinzipielle Vernachlässigung der Empirie zu attestieren. An Fraenkels Adresse gewandt war an diesen Vorwurf sehr leicht die Folgerung zu knüpfen, sie diene einer vorgängig beabsichtigten 
eine parlamentspragmatische Sicht anstelle der „Ideologie eines Parlamentsperfektionismus“ gemäß rousseauistischer Ratio; er wirbt mit Verve für eine historiographisch fundierte, komparativ-multikausale und interdisziplinäre Erklärung der Überlebenschancen notwendigerweise stets unfertiger Parlamente und damit stets defizitärer Demokratien. Entschieden tritt er - selbst ehedem, wie gesagt, klassenkämpferisch engagiert - sowohl zugespitzt idealistischem Parlamentarismusverständnis ${ }^{40}$ als auch jenem populär marxistisch-materialistisch zugerüsteten Parlamentsverständnis entgegen, wonach die Parlamente nichts als Verschleierungen des Klassenkampfes ${ }^{41}$ seien:

„Solange die Parlamente sich selber zu der, rationalen' Theorie der Demokratie bekannten, mußten sie ihre segensreiche Funktion, als clearing houses widerstreitender Partikularinteressen an der Erzeugung eines a posteriori Gemeinwohls mitzuwirken, verleugnen. Hierdurch veranlaßten sie nicht nur ihre Gegner, sondern auch die Theoretiker des Parlamentarismus ${ }^{42}$, sie mit falschem Maßstab zu messen. Sie forderten die Kritik der Wissenden und das Gemecker der Unwissenden geradezu heraus, die nicht müde wurden zu behaupten, die Parlamente seien Stätten des ,Parteiengezänks', ,Schwatzbuden' und Tummelplätze von ,Interessenhaufen'. Wenn sich die politische Elite einer Nation in der Ideologie eines Parlamentsperfektionismus verliert, kann es nicht Wunder nehmen, wenn sich im Massenbewußtsein das Gefühl der Parlamentsverdrossenheit breit macht. “43

Bei Berücksichtigung des von Fraenkel umfassend bereitgestellten analytischen Instrumentariums verwundert den Studierenden der heutigen Argumente zur „postparlamentarischen Demokratie“ 44 , gar zur „Postdemokratie“ ${ }^{45}$ manche Voraussetzungslosigkeit, um nicht zu sagen Ahnungslosigkeit der gegenwärtigen Diskussion. Erstaunlich in diesem Sinne ist auch, wie wenig sich sogar prominente Titelgeber dieser Diskussion gegen den Einwand möglicher Analogien zum Parlamentsverständnis à la Agnoli absichern ${ }^{46}$. Der Unterschied

Beschönigung der bundesrepublikanischen Wirklichkeit.

40 Wie von Karl Jaspers, nicht zuletzt unter dem Eindruck der „Tendenzen“ (S. 138 ff.) der sich anbahnenden ersten Großen Koalition der Bundesrepublik vorgetragen in seiner streitbewusst engagierten Schrift: Wohin treibt die Bundesrepublik?, München 1966. Siehe im POLITOLOGEN, 7. Jg. (1966), H. 20, S. 55 - 61 die kritische Auseinandersetzung von Uwe Thaysen mit dieser Veröffentlichung des Philosophen; siehe auch dessen Antwort darauf in: Karl Jaspers, Antwort. Zur Kritik meiner Schrift Wohin treibt die Bundesrepublik Deutschland, München 1967, S. 168 f.

41 Wie im selben Jahre mit Johannes Agnolis Involutionsthese (Fn. 32) intellektuell populär gemacht. Der von Agnoli konstatierte Klassenkampf wird seines Erachtens im Parlament und durch das Parlament insgesamt camoufliert und calmiert. Danach sind Parlamente nichts anderes als ,Transmissionsriemen der Entscheidungen politischer Oligarchien“, hier zitiert nach der EVA-Ausgabe der Transformationsschrift, a.a.O. (Fn. 32), S. 68.

42 Fraenkel verweist an dieser Stelle beispielhaft auf Gaetano Mosca, Die herrschende Klasse, München 1950, S. $2007 \mathrm{ff}$.

43 Ernst Fraenkel, Parlamentsverdrossenheit, a.a.O. (Fn. 18), S. 143 f.

44 Arthur Benz, Postparlamentarische Demokratie?, a.a.O. (Fn. 8).

45 Colin Crouch, Post-Democracy, Cambridge 2004.

46 Diese Verwunderung gilt vor allem Colin Crouch, der ansonsten sowohl komparativ und historiographisch als auch, ganz wie Agnoli, sozio-ökonomisch fokussiert ist. Crouch könnte / müsste von daher die deutsche Diskussion, auch und besonders die um Agnoli geführte Auseinandersetzung sehr wohl kennen. Die „privileged elites“ von Crouch, a.a.O., passim, wären jedenfalls umstandslos gleichzusetzen mit Agnolis „Herrschaftsgruppen der Produktionssphäre“, Faschismusdiskussion II, a.a.O. (Fn. 17), S. 33. Mehr noch: Was damals für Agnoli mit seiner Involutionsthese galt, gilt heute für Crouch: ,... while the forms of democracy remain fully in place ... politics and government are increasingly slipping back into the control of privileged elites in the manner 
zwischen Fraenkels und Agnolis Sicht der Parlamente besteht jedenfalls darin, dass Fraenkel am Parlamentarismus als „Referenzpunkt der Demokratie“47 festhält, während Agnoli sich ganz im Sinne Lenins, Demokratie auch „ohne Parlamente ... denken“48 kann und will. Welches ist der Schritt vom Parlamentsverdruss zum Parlamentsüberdruss? Und wie groß ist er, sofern er nicht essentieller, sondern nur gradueller Art sein sollte?

Die Gültigkeit der Fraenkelschen Aussage zur „Ideologie des Parlamentsperfektionismus“ erwies sich jedenfalls und erweist sich voraussichtlich auch weiterhin langlebiger, als Studentengenerationen es sind. Die studentischen Redakteure des POLITOLOGEN der Jahre 1965 ff. jedenfalls befanden sich um 1968 in ihren Abschlussexamen. Die von ihnen teils noch selbst mit ausgewählten Nachfolger für die Redaktion verschrieben sich verstärkt dem Geist der neuen „Studentenbewegung“ - entschieden stärker allemal, als dies der liberal und szientistisch orientierten Gründergeneration der „Zeitschrift für POLITOLOGIE“ lieb war, ganz zu schweigen von der Gründergeneration des POLITOLOGEN. Der seit 1969 neuen Redaktion kam es umgehend darauf an, die Gesellschaft nicht nur zu interpretieren, sondern gezielt zu verändern. Zunächst verschob sich das Spektrum der publizierten Analysen politisch deutlich nach „links“, wissenschaftlich in Richtung Marxismus und Frankfurter Schule. Für Autoren, die zuvor mit einem Totalitarismusverständnis gemäß Carl Joachim

characteristic of predemocratic times", a.a.O., S. 6. Arthur Benz, a.a.O. (Fn. 8), S. 219, bleibt behutsamer: Einerseits will er kein „Gegenmodell“ zum parlamentarischen Regierungssystem entwerfen, verweist dennoch andererseits hinsichtlich der von ihm beschriebenen „'schleichenden' Transformation der parlamentarischen Demokratie“ affirmativ darauf, dass „primär die Zuordnung der Elemente [dieser Transformation wie Verhandlung und Parteienwettbewerb] zu korrigieren“ sei. Diese neue Kombination zu erarbeiten, sei die „Aufgabe einer Demokratietheorie, die der realen Komplexität des modernen Staates angemessen" sei.

47 Emanuel Richter, Das Analysemuster der ,Postdemokratie'. Konzeptionelle Probleme und strategische Funktionen, in: Postdemokratie. Ein neuer Diskurs? Forschungsjounal NSB, 19. Jg. (2006), S. 33 ff. Die Autoren dieses Heftes, vor allem Hubertus Buchstein, Frank Nullmeier, Dirk Jörke und Emanuel Richter seien ausdrücklich ausgenommen von der hier ausgesprochenen Vermutung retrospektiver „Ahnungslosigkeit“. Siehe deren Beiträge im Forschungsjournal NSB, a.a.O. (Fn. 47). Siehe auch Hubertus Buchstein / Dirk Jörke, Das Unbehagen an der Demokratietheorie, a.a.O. (Fn. 5), mit ihren begriffsgeschichtlichen Nachweisen - nicht nur zu den Ursprüngen des Terminus „Postdemokratie“. Ihre demokratiegeschichtlich sowie demokratietheoretisch tiefer gehende und weiter gespannte Analyse begründet ebenso wohl ihre Skepsis gegenüber „poststrukturalistischen“ Modellen von Demokratie wie ihr Eintreten für eine normative „Dynamisierung" des Demokratiebegriffes anstelle seiner klassenkämpferischen wie seiner rationalistisch technokratischen Reduktion: „Jeglichem Bemühen um die definitive Verwirklichung von Demokratie steht entgegen, dass ihr ein konstitutives Äußeres, ein Moment von Nicht-Demokratie innewohnt, an dem sich das demokratische Versprechen kritisch abarbeiten muss. Ein dynamisierter Demokratiebegriff bezeichnet gegenüber den temporären Institutionalisierungen somit die prinzipielle Unabgeschlossenheit und Offenheit des demokratischen Projektes." (H. Buchstein I D. Jörke, S. 492 f. ) Emanuel Richter (S. 32) registriert „Tendenzen zu einer geradezu ostentativen Demokratieverdrossenheit ... einer buchstäblich schamlosen Demokratieverdrossenheit“.

48 W. I. Lenin, Staat und Revolution, in: W. I. Lenin, Ausgewählte Werke in zwei Bänden, Bd. II, Berlin 1952, S. 193: „Ohne Vertretungskörperschaften können wir uns eine Demokratie nicht denken, auch die proletarische nicht; ohne Parlamentarismus können und müssen wir sie uns denken ..." (Hervorhebung im Original). Exakt um das in diesem Aufsatz von Lenin propagierte Parlamentsverständnis ging es 1967/68 innerhalb der APO, unter anderem über die Beteiligung an Wahlen zu den Parlamenten und deren (möglicher) Instrumentalisierung zum Zwecke der Potenzierung des Klassenkonfliktes. Siehe dazu auch Gerhard A. Ritters Auseinandersetzung, a.a.O. (Fn. 33), mit Frank Deppe. 
Friedrich ${ }^{49}$ oder auch nur - dieses bereits deutlich relativierend - Peter Christian Ludz, oder mit einer Parlamentarismuslehre nach Fraenkel vertreten waren, gab es von nun an ebenso wenig eine Chance wie für Vertreter eines wertkonservativen oder liberalen Demokratieverständnisses, zum Beispiel Otto Heinrich von der Gablentz oder Richard Löwenthal.

Die neue Redaktion unter Leitung von Frank Stern, heute anerkannter Geschichts- und Kulturwissenschaftler in Israel, versuchte die Herausgeber des 9. Jahrganges (1968) durch eine Art Putsch zu suspendieren und die Zeitschrift für POLITOLOGIE für veränderte Zwecke zu usurpieren. Davon jedoch erfuhr der damals geschäftsführende Herausgeber Jürgen Falter, zum damaligen Zeitpunkt fern von Berlin, gerade noch rechtzeitig. Auf mehreren Umwegen und buchstäblich über Nacht beantragte er beim deutschen Patentamt wenigstens noch Schutz für die Titel DER POLITOLOGE und „Berliner Zeitschrift für POLITOLOGIE“ - mit Erfolg, so dass die geplante sozialistische Vierteljahresschrift wenigstens nicht unter diesen Titeln erscheinen konnte.

Unter Mitnahme der Abonnentenkartei und der (geringen) Kassenbestände machte sich sodann das selbsternannte neue „Redaktionskollektiv“ daran, ein Nachfolgeorgan mit gänzlich anderer Zielsetzung, die "Sozialistische Politik“ (SoPo), zu gründen. Den Verfassern dieser Zeilen war klar, dass sie auf keinerlei solidarischen (um nicht zu sagen: loyalen) Einsatz ihres damaligen Kollegen Agnoli im Redaktionsteam der Berliner Zeitschrift für Politikwissenschaft bauen konnten, „ihrer“ Zeitschrift gegen derlei Putschmanöver zum Überleben zu verhelfen. Gleichsam nahtlos setzte Johannes Agnoli seine fundamentale Parlamentarismuskritik in Heft 1 der SoPo fort. ${ }^{50}$ Im Editorial dieses Heftes hieß es:

„Die sozialistische Praxis in Hochschule und Gesellschaft muß stärker als bisher in unserer redaktionellen Arbeit zum Ausdruck kommen ... [Das Redaktionskollektiv] kann sich nur als Vermittler einer Lehr- und Forschungspraxis begreifen, die ihr Ziel in der radikalen Umwandlung der bestehenden Gesellschaft und der Entwicklung einer sozialistischen Alternative sieht. Die Formen dieser Veränderungen und ihre sozialistische Perspektive treten damit in den Vordergrund redaktioneller Arbeit. ... Wir verlassen damit bewußt den Standpunkt eines meinungsvermittelnden Aufklärers und nehmen dezidiert Stellung."

Neben Hella und Frank Stern zählte Bernard Langfermann zu den weiteren Mitgliedern des Redaktionskollektivs. Wie sich nach 1990 herausstellte, waren einige Mitglieder des Redaktionskomitees eng mit der Sozialistischen Einheitspartei Westberlin (SEW), dem Westberliner Ableger der Sozialistischen Einheitspartei Deutschlands (SED), verbunden. Ein noch des längeren aktives Redaktionsmitglied trat der SEW Anfang der 1970er Jahre auch offiziell bei. DDR-Widerständige von einst werfen diesem Mitarbeiter seit Jahren vor, der Leipziger Dissidentenszene als Stasi-Zuträger schlimmen Schaden, darunter mehrjährige Zuchthausstrafen, zugefügt zu haben ${ }^{51}$. Es sage niemand, die damalige Agitation in wissenschaftlichem Gewande sei praktisch bedeutungslos, nicht „aus der Nähe ... erlebbar“ gewesen.

49 Siehe Carl J. Friedrich, Grundzüge totalitärer Herrschaft, in: Der POLITOLOGE, 7. Jg. (1966), Nr. 20, S. $43-53$.

50 Johannes Agnoli, Zur Parlamentarismusdiskussion in der Bundesrepublik, in: SoPo, 1. Jg. (1969), H. 1, S. $1 \mathrm{ff}$.

51 Dietrich Koch, Das Verhör, 3 Bände, Dresden 2000/2001 passim, besonders Band I, S. 60 - 70 , den Abschnitt „Verrat aus dem Westen“; siehe auch ders. und Eckhard Koch, Denkschrift 2 für den Wiederaufbau der Leipziger Universitätskirche St. Pauli, http://wwischer.itrnet.com/wolfgang/koch/Denkschrift2-1-300.pdf (2. Mai 2007). 
Binnen kurzer Zeit wurde die SoPo offenkundig SED- und DDR-konform(er), was 1971 dazu führte, dass sich davon in einer Art roter Zellteilung eine weitere Zeitschrift abspaltete: das als „PROKLA“ bekannt gewordene Periodikum „Probleme des Klassenkampfs. Zeitschrift für politische Ökonomie und sozialistische Politik“. Michael Heinrich, geschäftsführender Redakteur schildert die Entwicklung wie folgt:

„Bis 1970 hatten in der ... SoPo noch verschiedene Fraktionen der ,Neuen Linken’ zusammengearbeitet. Mit der zunehmenden ,Parteiorientierung' war dies aber nicht mehr so ohne weiteres möglich; es sollte ja schließlich Klarheit herrschen, wo die ,führende Partei der Arbeiterklasse' zu suchen sei. Eine an der SEW/DKP orientierte Fraktion sicherte sich durch die Gründung einer GmbH das Eigentum an Namen und Vertrieb der SoPo, die dann allerdings nach wenigen Jahren einging. Der Mehrheit der Redaktionskonferenz, die mit dieser neuen Linie keineswegs einverstanden war, blieb nur noch der Auszug und die Neugründung einer eigenen Zeitschrift - der PROKLA. “52

Allerdings: Auch für diese Zeitschrift standen „der Bezug zur marxschen Kritik der politischen Ökonomie und die Orientierung auf eine im Entstehen begriffene revolutionäre Bewegung ... außer Zweifel" 53 . Als Ziel der PROKLA wurde im Editorial der ersten Nummer dementsprechend unmissverständlich klargestellt, mit den publizierten Analysen „Elemente einer Taktik des revolutionären Kampfes für die Gegenwart gewinnen“ zu wollen. „Das politische Ziel - Sozialismus - und der soziale Träger der gesellschaftlichen Veränderungen - die Arbeiterklasse - standen außer Frage. "54 Sowohl intellektuell als auch in ihrer Überlebensfähigkeit erwies sich die PROKLA als weitaus erfolgreicher - wenigstens darin eher in der Nachfolge des POLITOLOGEN stehend.

Der Sektion „Theorie“ der Deutschen Vereinigung für politische Wissenschaft hatte Agnoli im Oktober 1968 einen Forschungsbericht über den Stand der Parlamentarismusdiskussion vorgelegt, der im Eröffnungsheft der SoPo an erster Stelle veröffentlicht wurde ${ }^{55}$. Darin findet sich das seither sinngemäß - wenngleich nicht ironisch wie bei Agnoli - zum parlamentarischen Regierungssystem vielfach wiederholte Verdikt: „Leider muss der Referent gleich eingangs feststellen, daß es in dieser Hinsicht zwar viel zu erforschen, aber wenig zu berichten gibt. " $56 \mathrm{Zu}$ Ernst Fraenkel führt er darin ernsthaft aus:

„Die Scheu vor der Theorie hat teils ideologische, teils methodologische Gründe. In den Kontext der Ideologie gehört auch die sozialpsychologisch verständliche Wendung der älteren Politologie zur Affirmation der konstituierten Ordnung. Es handelt sich um einen eigentümlichen Gewissenskonflikt (deutlich bei E. Fraenkel, in geringerem Maße bei Löwenthal zu beobachten): um die Befürchtung, die kritische Haltung der Wissenschaft in der weimarer [sic] Zeit gegenüber den Problemen des Parlamentarismus, die damals geäußerten Zweifel, das Fehlen des vorbehaltlosen Bekenntnisses zum Verfassungsstaat und dessen Organen habe zum Untergang der ersten deutschen parlamentarischen Demokratie beigetragen. "

52 Michael Heinrich, PROKLA - oder wie ist heute eine linke, wissenschaftliche Zeitschrift möglich?, http://www.PROKLA.de/about frameset.htm (23. Januar 2007).

53 Ebenda.

54 Ebenda.

55 Johannes Agnoli, Zur Parlamentarismusdiskussion, a.a.O. (Fn. 50).

56 Ebenda, S. 1. Dieser eine Satz war zwar als eigenständiger Absatz deklariert, aber, typisch Agnoli, zugleich in Klammern gesetzt. Er liebte es, wonach ihm häufig war, lächerlich zu machen.

57 Ebenda, S. 1. 
Anders als in Großbritannien mit „Parliamentary Affairs“ und den USA mit „Congressional Quarterly“ gab es zu dieser Zeit in der Bundesrepublik kein Periodikum als Sammelstelle für die tatsächlich zunehmend vielfältigen empirischen Befunde zur parlamentarischen Regierungsweise - zumal nicht zur hiesigen -, auch keine Plattform zur theoretischen Diskussion des Parlamentarismus. Nicht zuletzt war es die akademische Popularität der Involutionsthese von Johannes Agnoli, besonders die darin vertretene Parlamentslehre, welche Winfried Steffani und Uwe Thaysen, beide Fraenkel-Schüler und seit 1968 an der Universität Hamburg, anspornte, an der Herausgabe einer Zeitschrift für Parlamentswissenschaft zu arbeiten ${ }^{58}$. Die Gründung der nun bald 40-jährigen ZParl stellte eine explizite Reaktion auf die außerparlamentarische und antiparlamentarische Stoßrichtung dar, wie sie von nun an in SoPo und PROKLA vertreten wurde. Personell, aber auch von der Grundausrichtung her lässt sich ein Bogen schlagen vom POLITOLOGEN der Jahre 1965 bis 1968 zur Zeitschrift für Parlamentsfragen. Es waren Studenten und Nachwuchswissenschaftler, die zu den Trägern der Etablierung und Ausbreitung der Politikwissenschaft in den 1960er Jahren zählten; nicht gerade wenige von ihnen gehören der in diesen Tagen und Jahren aus dem Amte scheidenden dritten Generation der akademischen Politikwissenschaft an, zu deren Kernbereichen nach wie vor die Parlamentsforschung und die in dieser Zeitschrift, der ZParl, nicht zufällig massiv dokumentierten Teildisziplinen der Wahl- und der Parteienforschung gehören.

Aus hierzulande leidvoller Erfahrung ist die Wirkungsgeschichte politischer Ideologien und Theoreme einschließlich ihrer Begriffe in Rechnung zu stellen: Heute wie damals ist nach der Referenzbasis ${ }^{59}$ von Demokratie und Demokratietheorie zu fragen. Mit welchen Klassifizierungen ist die Bestandsfähigkeit von Demokratie zu fördern, um nicht zu sagen: zu behaupten? Bezogen auf die gegenwärtige Diskussion um „Postdemokratie“ stellen sich

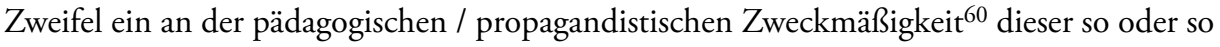
- rückwärts- oder zukunftsgewandt - pejorativen Etikettierung. Bezogen auf die wissenschaftliche Tauglichkeit des Begriffes „Postdemokratie“ ist mindestens Skepsis angebracht, ob dieser als (empirischer) „Beschreibungsterminus“ gleichgesetzt werden darf mit einer ungeklärten (normativen) „Programmvokabel“61. Dasselbe gilt für „postparlamentarische Demokratie" und deren Variationen.

58 Siehe dazu auch Wilhelm Bleek, a.a.O. (Fn. 12), S. 295. Aus der Sicht der Verfasser ist es erfreulich, eine deutliche Rückbesinnung ihrer Disziplin auf Ernst Fraenkel zu beobachten. Wir sehen darin die angemessene Würdigung eines zwar fragmentiert erschienenen, aber bedeutsamen deutschen Beitrages zur Geschichte der Parlamente wie zur Parlamentarismustheorie. Erfreulich ist auch die Ankündigung des Nomos-Verlages, dass der Band V der Gesammelten Schriften von Fraenkel demnächst erscheinen wird. Denn die in diesem Band versammelten Beiträge lassen die Konsistenz - eine bemerkenswerte intellektuelle Disziplin - erkennen, mit welcher Fraenkel seine Parlamentarismustheorie Mosaik um Mosaik zusammenfügte.

59 Ein Terminus, den wir in Anlehnung an den von Emanuel Richter, a.a.O. (Fn. 49), gewählten „Referenzpunkt“ empfehlen - sicherlich ohne Abstriche oder Veränderung des von Richter gemeinten Inhaltes.

60 Dazu jüngst Gerhard Himmelmann, Leitbild und Demokratieerziehung. Vorläufer, Begleitstudien und internationale Ansätze zum Demokratie-Lernen, Schwalbach/Ts. 2006, besonders S. 70 ff.

61 Zur hier aufgenommenen Begrifflichkeit siehe Hubertus Buchstein / Frank Nullmeier, Zur Postdemokratie-Debatte, in: Forschungsjounal NSB, 19. Jg. (2006), S. 20. 